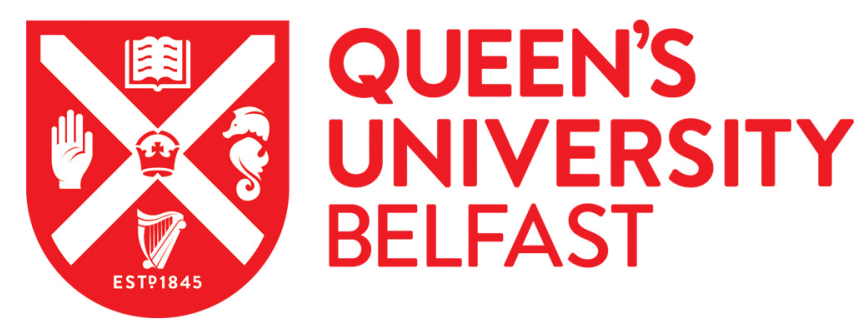

\title{
Sparse Heteroscedastic Multiple Spline Regression Models for Wind Turbine Power Curve Modeling
}

\author{
Wang, Y., Li, Y., ZOU, R., Foley, A. M., Alkez, D., Song, D., Hu, Q., \& Srinivasan, D. (2021). Sparse \\ Heteroscedastic Multiple Spline Regression Models for Wind Turbine Power Curve Modeling. IEEE Transactions \\ on Sustainable Energy, 12(1), 191-201. https://doi.org/10.1109/tste.2020.2988683
}

Published in:
IEEE Transactions on Sustainable Energy

Document Version:

Peer reviewed version

Queen's University Belfast - Research Portal:

Link to publication record in Queen's University Belfast Research Portal

Publisher rights

(c) 2020 IEEE.

This work is made available online in accordance with the publisher's policies. Please refer to any applicable terms of use of the publisher.

\section{General rights}

Copyright for the publications made accessible via the Queen's University Belfast Research Portal is retained by the author(s) and / or other copyright owners and it is a condition of accessing these publications that users recognise and abide by the legal requirements associated with these rights.

Take down policy

The Research Portal is Queen's institutional repository that provides access to Queen's research output. Every effort has been made to ensure that content in the Research Portal does not infringe any person's rights, or applicable UK laws. If you discover content in the Research Portal that you believe breaches copyright or violates any law, please contact openaccess@qub.ac.uk. 


\title{
Sparse Heteroscedastic Multiple Spline Regression Models for Wind Turbine Power Curve Modeling
}

\author{
Yun Wang, Yifen Li, Runmin Zou, Aoife M. Foley, Member, IEEE, Dlzar Al Kez, Dongran Song, Qinghua \\ $\mathrm{Hu}$, Senior Member, IEEE and Dipti Srinivasan, Fellow, IEEE
}

\begin{abstract}
An accurate wind turbine power curve (WTPC) plays a vital role in wind power forecasting and wind turbine condition monitoring. There are two major shortcomings of current WTPC models that prevent more accurate WTPC estimation, limited nonlinear fitting ability and the lack of indepth understanding of the complex characteristics of WTPC. This paper proposes two novel regression models to overcome these two disadvantages simultaneously. First, they make use of multiple spline regression models (MSRM) with different basis functions and different numbers of knots to describe the complex nonlinear relationship between wind speed and wind power. Moreover, sparse prior distributions help avoid the adverse effects of redundant mapping features and useless basis functions on the model performance. Second, they embed the heteroscedasticity of WTPC modeling into MSRM based on Gaussian and Student's $t$-distributions, respectively. Finally, two sparse heteroscedastic MSRM with Gaussian and Student's $t$ distributions will be constructed and named as SHMSRM-G and SHMSRM-T, respectively. We compare the proposed models with fifteen benchmark models, and find that they can generate more accurate WTPCs than the others in different seasons and different wind farms. Thus, it is important to consider the complex nonlinear fitting ability and heteroscedasticity together in constructing accurate WTPC models.
\end{abstract}

Index Terms-Power curve modeling, multiple spline regression models, sparsity, heteroscedasticity, variational Bayesian.

\section{INTRODUCTION}

$\mathbf{R}$ ECENTLY, the development and utilization of renewable energy has become a common way to solve the problem of energy shortage and environmental pollution worldwide [1]. Wind energy, as a clean, environmentally friendly, inexhaustible and inexpensive renewable energy, has attracted more and more attention [2]. An accurate WTPC plays a vital role in many applications of wind power [3].

Usually, wind turbine manufacturers will provide theoretical WTPCs for their own wind turbine, which describes the performance of a wind turbine at a particular air density [4]. However, the air density varies with the change of time

This work is supported by National Natural Science Foundation of China under Grants 61925602 and 61732011. (Corresponding author: Runmin Zou)

Y. Wang, R. Zou and D. Song are with the School of Automation, Central South University, Changsha, China (e-mail: wangyun19@csu.edu.cn, rmzou@csu.edu.cn, humble_szy@163.com.).

Y. Li is with the School of Traffic and Transportation Engineering, Central South University, Changsha, China (e-mail: yifen.li@csu.edu.cn).

Q. Hu is with the College of Intelligence and Computing, Tianjin University, China (e-mail: huqinghua@tju.edu.cn).

A. Foley and D. Alkez are with the School of Mechanical and Aerospace Engineering, Queens University Belfast, U.K (e-mail: a.foley@qub.ac.uk, dalkez01@qub.ac.uk).

D. Srinivasan is with the Department of Electrical and Computer Engineering, National University of Singapore, Singapore (e-mail: dipti@nus.edu.sg). and geographic location. As a result, the same wind turbine will also perform differently during different seasons and at different wind farms. It is essential to model the actual WTPCs in practice. In literature, according to the modeling theory, the deterministic WTPC models can be divided into two categories, curve fitting models and artificial intelligence (AI)-based models [3].

Curve fitting models, which are usually S-shaped, can be grouped into two parts: the segmented curve fitting models and the integrated curve fitting models [3]. Examples of the former contain linearized segmented model [5], polynomial model [6], Sigmoid function-based [7] and Gaussian cumulative distribution function-based [8] power curves. The popular models in the latter are three-parameter logistic function (3-PLF) [9], four-parameter logistic function (4-PLF) [10], five-parameter logistic function (5-PLF) [10], six-parameter logistic function (6-PLF) [11] and the modified hyperbolic tangent (MHTan) [12]. As for AI-based models, they can learn the complex nonlinear relationship between wind speed and wind power automatically. The popular ones are support vector machine (SVM) [13], Gaussian process (GP) [14], extreme learning machine (ELM) [15], spline regression-based models [1] [4], adaptive neuro-fuzzy inference system (ANFIS) [16], monotonic regression [17] and copula model [18] etc.

Currently, two strategies are usually considered to obtain more accurate WTPCs. First, improving the quality of raw wind data. In the open environment, the collected data contain many uncertainties, such as outliers and missing data, which will affect the process of model training [19]. A man-made approach was designed to detect outliers in wind data [4]. Three-sigma rule [13] and K-means clustering [20] were utilized to filter the original wind data. In [19], quartile method and density-based clustering were employed to process different types of outliers. Second, developing novel WTPC models by considering the task characteristics. It was reported that WTPC modeling was characterized by heteroscedasticity and non-Gaussian error distribution. Based on the above findings, Wang et al. proposed heteroscedastic spline regression model (HSRM) [4], robust spline regression mode (RSRM) [4], asymmetric spline regression with mixture of asymmetric Gaussian distributions (MoAG-ASR) [1] and asymmetric spline regression with mixture of asymmetric exponential power distributions (MoAEP-ASR) [1]. Also, Rogers et al. utilized a heteroscedastic GP regression to model WTPCs [21].

In practice, due to the complexity of climate and environment, the actual WTPCs will be also very complex. In this case, they cannot be modelled by single models 
comprehensively. So, developing novel WTPC models with superior nonlinear fitting ability is also a useful strategy for generating accurate WTPCs. This paper takes account of some spline regression models with different spline basis functions (SBFs) and different numbers of knots in describing the complex nonlinear WTPCs. Thus, a framework of multiple spline regression models (MSRM) was constructed. However, some redundant mapping features and SBFs may have adverse effects on the regression performance of MSRM. To solve this problem, sparse prior distributions were given to the regression coefficients and the weights of SBFs to get sparse solutions of model parameters in MSRM. Besides, based on Gaussian distribution and Student's $t$-distribution, the characteristic of WTPC modeling, heteroscedasticity, was also considered in MSRM, thus resulting in two sparse heteroscedastic MSRM, SHMSRM-G and SHMSRM-T, respectively.

In order to optimize the proposed models, two methods, expectation maximization (EM) and variational Bayesian (VB), come into our mind. EM is a partially non-Bayesian, maximum likelihood method [22]. It gives probability distributions over the latent variables together with point estimates for the other parameters [22]. The computed point estimates are the modes of these parameters; no other information is available. As to VB, it estimates the actual posterior distributions of all variables, both parameters and latent variables. When point estimates need to be derived, generally the mean is used rather than the mode, as is normal in Bayesian inference [23]. Due to more information is provided for model parameters, VB will be used to optimize the proposed models. The main contributions of the paper can be summarized as follows.

- A framework of MSRM was constructed to enhance the nonlinear fitting ability of the proposed models in modeling the actual complex WTPCs.

- Sparse prior distributions were used to get the sparse model solutions, which help avoid the bad effects of the redundant mapping features and useless SBFs.

- The heteroscedasticity of WTPC modeling was embedded into the proposed models based on Gaussian distribution and Student's $t$-distribution, respectively.

The rest of the paper is organized as follows. Section II introduces the spline regression model (SRM) briefly. The two proposed models are described in detail in Section III. Section IV presents the processes of WTPC modeling. Section $\mathrm{V}$ shows the experiments and the corresponding experimental analysis, while Section VI concludes the whole paper.

\section{SPLINE REGRESSION MODEL}

SRM has been widely used in WTPC modeling due to its superior nonlinear fitting ability [1] [4] [24]. In WTPC modeling, supposing $x_{i}$ and $y_{i}$ denote wind speed and the corresponding power output, respectively. Given $N$ training samples $\left\{x_{i}, y_{i}\right\}_{i=1, \cdots, N}$, a general SRM can be expressed as

$$
y_{i}=f_{\text {basis }}\left(x_{i}\right)+\varepsilon_{i}, \quad(i=1, \cdots, N)
$$

where $\varepsilon_{i}$ is the regression error, $f_{\text {basis }}(\cdot)$ is the estimated function related with a SBF. Equation (1) can be expressed in a matrix form,

$$
\mathbf{Y}=\mathbf{H} \boldsymbol{\beta}+\varepsilon
$$

where $\mathbf{Y}=\left[y_{1}, \cdots, y_{N}\right]^{\top} \in \mathbb{R}^{N}$ is the vector of output, $\varepsilon=\left[\varepsilon_{1}, \cdots, \varepsilon_{N}\right]^{\top} \in \mathbb{R}^{N}$ is the vector of regression error, $\boldsymbol{\beta}=\left[\beta_{1}, \cdots, \beta_{M}\right]^{\top} \in \mathbb{R}^{M}$ is the vector of regression coefficients, $\boldsymbol{h}_{m} \in \mathbb{R}^{N}$ is the $m$ th mapping feature generated by the selected $f_{\text {basis }}(\cdot), \mathbf{H}=\left[\boldsymbol{h}_{1}, \cdots, \boldsymbol{h}_{M}\right] \in \mathbb{R}^{N \times M}$ is the matrix of all mapping features, $M$ is the number of mapping features, which are related with the number of knots set in $f_{\text {basis }}(\cdot)$ [24].

In literature, two popular SBFs, truncated power basis and B-spline basis, are widely used to construct a SRM. Their expressions can be referred to [1], [4] and [24]. The least squares solution for (2) is $\hat{\boldsymbol{\beta}}=\left(\mathbf{H}^{\top} \mathbf{H}\right)^{-1} \mathbf{H Y}$ [24].

\section{SPARSE HETEROSCEDASTIC MULTIPLE SPLINE REGRESSION MODELS}

There are three major drawbacks of the original SRM: (a) sometimes the performance of SRM is sensitive to the number of knots, and the optimal one varies with the task [24]; (b) the SRM with single SBF has the limited ability in describing the complex nonlinear relationship; (c) the underlying assumption in SRM is not consistent with the characteristics of the observed task, resulting in the biased results. To solve these drawbacks, this paper proposed two types of sparse heteroscedastic MSRM, SHMSRM-G and SHMSRM-T.

The proposed models take advantage of several spline regression models with different types of SBFs and various numbers of knots, and the weight of each model and regression coefficients are optimized automatically by sparse Bayesian learning. So, they can not only describe the complex nonlinear relationship with MSRM, but also avoid the adverse effects of redundant mapping features and useless SBFs automatically, thus overcoming the first and second drawbacks of SRM. For a wind turbine, at the same wind speed, different environmental conditions may result in different power output. So, it can be found that the variance of wind power will vary with wind speed. The errors of WTPC modeling will be heteroscedastic through analysing [4]. The proposed models employed the Gaussian distribution-based and Student's $t$-distributionbased heteroscedastic error assumptions to replace the original Gaussian error assumption, respectively, thus overcoming the third drawback of SRM. The framework of MSRM and the proposed models are introduced in detail below.

\section{A. Framework of multiple spline regression models (MSRM)}

Given the input data $\left\{x_{i}\right\}_{i=1, \cdots, N}, K$ SBF matrices $\mathbf{H}_{1}, \mathbf{H}_{2}, \cdots, \mathbf{H}_{K}$ can be calculated with different numbers of knots and different SBFs. The numbers of mapping features in these matrices are $M_{1}, M_{2}, \cdots, M_{K}$, respectively. The size of $\mathbf{H}_{i}$ may be different from $\mathbf{H}_{j}$ when $i \neq j$. They can be transformed into the same size by (3)

$$
\widetilde{\mathbf{H}}_{k}=\left[\mathbf{H}_{k}, \mathbf{0}_{N \times\left(\widetilde{M}-M_{k}\right)}\right], k=1, \cdots, K
$$

where $\widetilde{\mathbf{H}} \in \mathbb{R}^{N \times \widetilde{M}}, \mathbf{0}_{N \times\left(\widetilde{M}-M_{k}\right)}$ is the $N \times\left(\widetilde{M}-M_{k}\right)$ zero matrix, and $\widetilde{M}=\max \left\{M_{1}, M_{2}, \cdots, M_{K}\right\}$. So, in order to avoid selecting optimal mapping features and SBFs, and to 
take advantages of different single SRMs, the framework of MSRM is proposed and given by

$$
y_{i}=\boldsymbol{\omega}^{\top}\left[\left(\widetilde{\mathbf{H}}_{1}^{(i, \cdot)}\right)^{\top}, \cdots,\left(\widetilde{\mathbf{H}}_{K}^{(i, \cdot)}\right)^{\top}\right]^{\top} \boldsymbol{\beta}+\varepsilon_{i}
$$

where $\boldsymbol{\omega}=\left[\omega_{1}, \cdots, \omega_{K}\right]^{\top} \in \mathbb{R}^{K}$ is the weight vector of different SBF matrices, $\boldsymbol{\beta}=\left[\beta_{1} \cdots, \beta_{\widetilde{M}}\right]^{\top} \in \mathbb{R}^{\widetilde{M}}$ is the vector of regression coefficients, $\widetilde{\mathbf{H}}_{k}^{(i, \cdot)}$ is the $i$ th row of $\widetilde{\mathbf{H}}_{k}$. The matrix form of (4) is

$$
\mathbf{Y}=\widetilde{\mathbf{H}}_{\boldsymbol{\omega}} \boldsymbol{\beta}+\boldsymbol{\varepsilon}
$$

where $\widetilde{\mathbf{H}}_{\boldsymbol{\omega}}=\sum_{k=1}^{K} \omega_{k} \widetilde{\mathbf{H}}_{k} \in \mathbb{R}^{N \times \widetilde{M}}$.

Within the framework of MSRM, $\boldsymbol{\beta}$ and $\boldsymbol{\omega}$ can be learnt simultaneously with the training data. In the following subsection, sparse heteroscedastic models will be developed based on MSRM from Bayesian perspective.

\section{B. Sparse heteroscedastic multiple spline regression models with Gaussian distribution (SHMSRM-G)}

To develop the proposed model, the prior distributions of all parameters in SHMSRM-G are given and the corresponding posterior distributions are also derived in detail as follows.

1) Prior distributions of all parameters in SHMSRM-G: A sparse representation has several advantages, e.g. over-training is often avoided [25] and inferred sparse coefficients also often have biological/physical meaning, of interest for model interpretation [26]. In this paper, sparse solutions for the model weight vector $(\boldsymbol{\omega})$ and the regression coefficients $(\boldsymbol{\beta})$ can help reduce the adverse effects of some useless SBFs and mapping features. So, considering the above benefits, it is essential to build a sparse MSRM.

Generally, a sparse model can be obtained through prior distributions that have fat tails and sharp peaks at zero [27], [28]. Such priors are also denoted as sparsity-inducing priors. So, based on sparse Bayesian learning, $p\left(\beta_{m}\right)$ and $p\left(\varrho_{m}\right)$ are given a Gaussian distribution $\mathcal{N}(\cdot)$ and a Gamma distribution $\mathcal{G}(\cdot)$ with two hyperparameters $b_{0}, c_{0}$, respectively [29],

$$
p\left(\beta_{m}\right)=\mathcal{N}\left(\beta_{m} \mid 0, \varrho_{m}^{-1}\right), p\left(\varrho_{m}\right)=\mathcal{G}\left(\varrho_{m} \mid b_{0}, c_{0}\right) .
$$

Similar to $\boldsymbol{\beta}$, the weight vector of all SBF matrices, $\boldsymbol{\omega}$, should also have sparse characteristic to reduce the adverse effects of some unreasonable numbers of knots and SBFs on the final regression results. Thus,

$$
p\left(\omega_{k}\right)=\mathcal{N}\left(\omega_{k} \mid 0, \eta_{k}^{-1}\right), p\left(\eta_{k}\right)=\mathcal{G}\left(\eta_{k} \mid d_{0}, e_{0}\right) .
$$

where $d_{0}, e_{0}$ are two hyperparameters. In certain applications, due to the complex data collection environment and different collection devices, or the various source of data generation, the error often does not obey Gaussian distributions. For example, it was reported that the error distribution of WTPC modeling was heteroscedastic. To overcome the inconsistence between the error assumption in the model and the real error characteristic in practice, and considering that Central Limit Theorem makes Gaussian distribution a good choice for modeling the regression errors or noise [30], the error term $\varepsilon_{i}$ in MSRM is given by the following Gaussian distribution,

$$
p\left(\varepsilon_{i}\right)=\mathcal{N}\left(\varepsilon_{i} \mid 0, \gamma_{i}^{-1}\right) .
$$

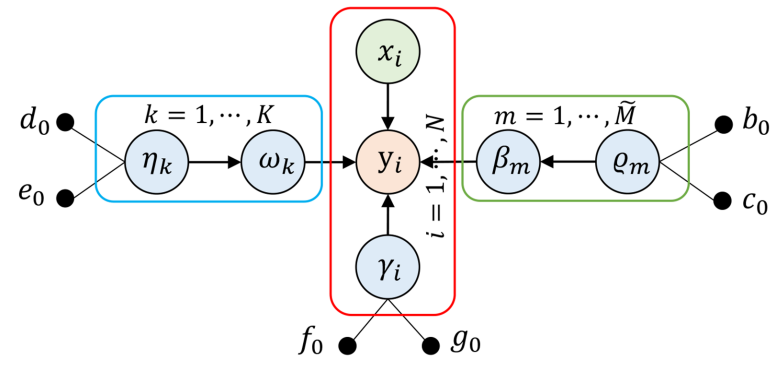

Fig. 1. Hierarchic priors for all parameters in SHMSRM-G.

From (8), for different samples, the error obey different Gaussian distributions. It means that the error is heteroscedastic, which meets the task characteristic of WTPC modeling. To form a full probability model, the prior distribution of $\gamma_{i}$ is assumed to obey a Gamma distribution with the hyperparameters $f_{0}, g_{0}$,

$$
p\left(\gamma_{i}\right)=\mathcal{G}\left(\gamma_{i} \mid f_{0}, g_{0}\right)
$$

According to (8), the likelihood of the observation $\mathbf{Y}$ can be expressed as

$$
p(\mathbf{Y})=\prod_{i=1}^{N} \mathcal{N}\left(y_{i} \mid \widetilde{\mathbf{H}}_{\boldsymbol{\omega}}^{(i, \cdot)} \boldsymbol{\beta}, \gamma_{i}^{-1}\right)=\prod_{i=1}^{N} \mathcal{N}\left(y_{i} \mid \boldsymbol{\omega}^{\top} \widetilde{\mathbf{H}}^{(i, \cdot)} \boldsymbol{\beta}, \gamma_{i}^{-1}\right)
$$

where $\widetilde{\mathbf{H}}_{\boldsymbol{\omega}}^{(i, \cdot)}$ denotes the $i$ th row of the matrix $\widetilde{\mathbf{H}}_{\boldsymbol{\omega}}$ and $\widetilde{\mathbf{H}}^{(i, \cdot)}=$ $\left[\left(\widetilde{\mathbf{H}}_{1}^{(i, \cdot)}\right)^{\top}, \cdots,\left(\widetilde{\mathbf{H}}_{K}^{(i, \cdot)}\right)^{\top}\right]^{\top} \in \mathbb{R}^{K \times \widetilde{M}}$.

Finally, the Bayesian model for SHMSRM-G can be constructed with the prior distributions from (6) to (10). The joint probability density function of the observed data $\mathbf{Y}$ and the corresponding parameters $\Theta$ is given by

$$
p(\mathbf{Y}, \boldsymbol{\Theta})=p(\mathbf{Y}) \prod_{m=1}^{\widetilde{M}} p\left(\varrho_{m}\right) p\left(\beta_{m}\right) \prod_{k=1}^{K} p\left(\eta_{k}\right) p\left(\omega_{k}\right) \prod_{i=1}^{N} p\left(\gamma_{i}\right)
$$

where $\varrho=\left\{\varrho_{1}, \cdots, \varrho_{\widetilde{M}}\right\}, \boldsymbol{\eta}=\left\{\eta_{1}, \cdots, \eta_{K}\right\}, \quad \gamma=$ $\left\{\gamma_{1}, \cdots, \gamma_{N}\right\}$, and $\boldsymbol{\Theta}=\{\boldsymbol{\varrho}, \boldsymbol{\beta}, \boldsymbol{\omega}, \boldsymbol{\eta}, \boldsymbol{\gamma}\}$. Then, our goal turns to infer the posteriors of all involved variables in (11).

2) Posterior distributions of all parameters in SHMSRM-G: In this paper, VB is employed to estimate all parameters in the joint probability $p(\mathbf{Y}, \boldsymbol{\Theta})$. The posterior distribution $q(\boldsymbol{\Theta})$ is supposed to approximate $p(\mathbf{Y}, \boldsymbol{\Theta})$, the factorized form of $q(\boldsymbol{\Theta})$ is expressed as

$$
q(\boldsymbol{\Theta})=q(\boldsymbol{\omega}) q(\boldsymbol{\eta}) q(\boldsymbol{\beta}) q(\varrho) q(\boldsymbol{\gamma}) .
$$

According to the theory of VB, the posteriors of all model parameters can be calculated as follows.

First, taking the expectation of the logarithmic joint probability $\log p(\mathbf{Y}, \boldsymbol{\Theta})$ with respect to $\boldsymbol{\Theta}$ except for $\boldsymbol{\beta}$, it can be concluded that the posterior of $\boldsymbol{\beta}, q(\boldsymbol{\beta})$, is a multivariate Gaussian distribution

$$
q(\boldsymbol{\beta})=\mathcal{N}\left(\boldsymbol{\beta} \mid \mu_{\boldsymbol{\beta}}, \boldsymbol{\Sigma}_{\boldsymbol{\beta}}\right)
$$


with the mean $\mu_{\boldsymbol{\beta}}$ and covariance $\Sigma_{\boldsymbol{\beta}}$ updated by

$$
\begin{gathered}
\left.\boldsymbol{\Sigma}_{\boldsymbol{\beta}}=\left[\sum_{i=1}^{N}\left\langle\gamma_{i}\right\rangle \widetilde{\mathbf{H}}^{(i, \cdot) \top}\left\langle\boldsymbol{\omega} \boldsymbol{\omega}^{\top}\right\rangle \widetilde{\mathbf{H}}^{(i, \cdot)}+\mathbf{A}\right)\right]^{-1} \\
\boldsymbol{\mu}_{\boldsymbol{\beta}}=\sum_{i=1}^{N}\left\langle\gamma_{i}\right\rangle y_{i} \boldsymbol{\Sigma}_{\boldsymbol{\beta}} \widetilde{\mathbf{H}}^{(i, \cdot) \top}\langle\boldsymbol{\omega}\rangle
\end{gathered}
$$

where $\mathbf{A}=\operatorname{diag}\left(\left\langle\varrho_{1}\right\rangle, \cdots,\left\langle\varrho_{\widetilde{M}}\right\rangle\right),\langle\cdot\rangle$ means the expectation calculation, and the function $\operatorname{diag}(\cdot)$ is used to make a diagonal matrix.

Subsequently, by calculating the expectation of $\log p(\mathbf{Y}, \mathbf{\Theta})$ with respect to $\Theta$ except for $\varrho$, it can be found that the posterior of $\varrho_{m}$ is a Gamma distribution, namely

$$
q\left(\varrho_{m}\right)=\mathcal{G}\left(\varrho_{m} \mid b_{\varrho_{m}}, c_{\varrho_{m}}\right)
$$

with hyperparameters $b_{\varrho_{m}}, c_{\varrho_{m}}$ updated by

$$
b_{\varrho_{m}}=b_{0}+\frac{1}{2}, c_{\varrho_{m}}=c_{0}+\frac{1}{2}\left\langle\beta_{m}^{2}\right\rangle .
$$

By calculating $\langle\log p(\mathbf{Y}, \boldsymbol{\Theta})\rangle$ with respect to $\boldsymbol{\Theta}$ except for $\boldsymbol{\omega}$, it can be inferred that $q\left(\omega_{k}\right)$ is a multivariate Gaussian distribution,

$$
q(\boldsymbol{\omega})=\mathcal{N}\left(\boldsymbol{\omega} \mid \boldsymbol{\mu}_{\boldsymbol{\omega}}, \boldsymbol{\Sigma}_{\boldsymbol{\omega}}\right)
$$

with the mean $\boldsymbol{\mu}_{\boldsymbol{\omega}}$ and covariance $\boldsymbol{\Sigma}_{\boldsymbol{\omega}}$ updated by

$$
\begin{gathered}
\boldsymbol{\Sigma}_{\boldsymbol{\omega}}=\left[\sum_{i=1}^{N}\left\langle\gamma_{i}\right\rangle \widetilde{\mathbf{H}}^{(i, \cdot)}\left\langle\boldsymbol{\beta} \boldsymbol{\beta}^{\top}\right\rangle \widetilde{\mathbf{H}}^{(i, \cdot) \top}+\mathbf{B}\right]^{-1} \\
\boldsymbol{\mu}_{\boldsymbol{\omega}}=\sum_{i=1}^{N}\left\langle\gamma_{i}\right\rangle y_{i} \boldsymbol{\Sigma}_{\boldsymbol{\omega}} \widetilde{\mathbf{H}}^{(i, \cdot)}\langle\boldsymbol{\beta}\rangle
\end{gathered}
$$

where $\mathbf{B}=\operatorname{diag}\left(\left\langle\eta_{1}\right\rangle, \cdots,\left\langle\eta_{K}\right\rangle\right)$.

For the parameter $\boldsymbol{\eta}$, the posterior $q\left(\eta_{k}\right)$ is a Gamma distribution parameterized with $g_{\eta_{k}}$ and $h_{\eta_{k}}$,

$$
\begin{gathered}
q\left(\eta_{k}\right)=\mathcal{G}\left(\eta_{k} \mid d_{\eta_{k}}, e_{\eta_{k}}\right), \\
d_{\eta_{k}}=d_{0}+\frac{1}{2}, e_{\eta_{k}}=e_{0}+\frac{1}{2}\left\langle\omega_{k}^{2}\right\rangle .
\end{gathered}
$$

Similarly, for the posterior of $\gamma_{i}$, it obeys a Gamma distribution,

$$
\begin{gathered}
q\left(\gamma_{i}\right)=\mathcal{G}\left(\gamma_{i} \mid f_{\gamma_{i}}, g_{\gamma_{i}}\right), \\
f_{\gamma_{i}}=f_{0}+\frac{1}{2}, \\
g_{\gamma_{i}}=g_{0}+\frac{1}{2}\left\langle\left(y_{i}-\omega^{\top} \widetilde{\mathbf{H}}^{(i, \cdot)} \boldsymbol{\beta}\right)^{2}\right\rangle .
\end{gathered}
$$

The posterior distributions of all parameters in SHMSRM-G are inferred and presented from (13) to (20). The optimization procedure of SHMSRM-G is summarized in Algorithm 1.

\section{Sparse heteroscedastic multiple spline regression models with Student's t-distribution (SHMSRM-T)}

Different from SHMSRM-G, the error is assumed to obey a Student's $t$-distribution, thus resulting in a novel regression model, namely SHMSRM-T. The main reasons for the utilization of Student's $t$-distribution error assumption are presented as follows. First, Student's $t$-distribution is more general than Gaussian distribution. Specifically, when the degree of

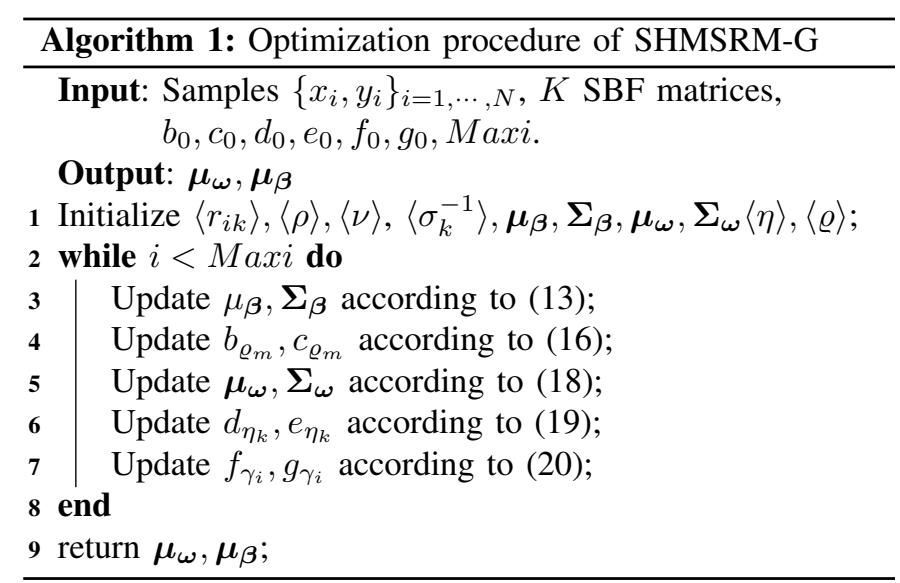

freedom in the probability density function of Student's $t$ distribution is equal to 1 , it reduces to a Cauchy distribution; it approaches a Gaussian distribution when the degree of freedom tends to infinity [31]. Second, it is a heavy-tailed distribution, which is more robust than Gaussian distribution [32]. The priors and posteriors of all model parameters are described as follows.

1) Prior distributions of all parameters in SHMSRM-T: Generally, the probability density function of a Student's $t$ distribution with zero mean and $\nu$ degree of freedom can be expressed as

$$
\mathcal{T}(\varepsilon \mid 0, \sigma, \nu)=\frac{\Gamma\left(\frac{\nu+1}{2}\right)}{\Gamma\left(\frac{\nu}{2}\right) \sqrt{\nu \pi} \sigma}\left(1+\frac{\varepsilon^{2}}{\nu \sigma^{2}}\right)^{-\frac{\nu+1}{2}}
$$

where $\sigma$ is the scale parameter. In order to describe the heteroscedasticity of the regression errors, they are assumed to obey Student's $t$-distributions, whose parameters vary with the sample,

$$
p\left(\varepsilon_{i}\right)=\mathcal{T}\left(\varepsilon_{i} \mid 0, \sigma_{i}, \nu_{i}\right) .
$$

It is reported that Student's $t$-distribution is a type of scale mixture of Gaussians [32]. So, it can be rewritten as [32], [33]

$$
\mathcal{T}(\varepsilon \mid 0, \sigma, \nu)=\int_{0}^{\infty} \mathcal{N}\left(\varepsilon \mid 0, \phi^{-1} \sigma\right) \mathcal{G}\left(\phi \mid \frac{\nu}{2}, \frac{\nu}{2}\right) d \phi .
$$

According to (23), a two-level generative model can be used to represent the heteroscedastic Student's $t$-error distribution in (22), namely

$$
p\left(\varepsilon_{i}\right)=\mathcal{N}\left(\varepsilon_{i} \mid 0, \phi_{i}^{-1} \sigma_{i}\right), p\left(\phi_{i}\right)=\mathcal{G}\left(\phi_{i} \mid \frac{\nu_{i}}{2}, \frac{\nu_{i}}{2}\right) .
$$

Thus, the likelihood function $p(\mathbf{Y})$ for the observed data is

$$
p(\mathbf{Y})=\prod_{i=1}^{N} \mathcal{N}\left(y_{i} \mid \boldsymbol{\omega}^{\top} \widetilde{\mathbf{H}}^{(i, \cdot)} \boldsymbol{\beta}, \phi_{i}^{-1} \sigma_{i}\right)
$$

To complete a full probability model for SHMSRM-T, the scale parameter $\sigma_{i}$ and the degrees of freedom $\nu_{i}$ are assumed to obey an inverse Gamma distribution and a Gamma distribution, respectively,

$$
p\left(\sigma_{i}\right)=\mathcal{I} \mathcal{G}\left(\sigma_{i} \mid h_{0}, i_{0}\right), p\left(\nu_{i}\right)=\mathcal{G}\left(\nu_{i} \mid r_{0}, s_{0}\right)
$$

where $h_{0}, i_{0}, r_{0}, s_{0}$ are hyperparameters. 


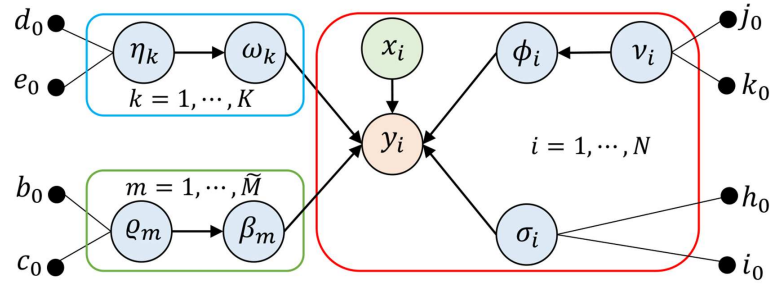

Fig. 2. Hierarchic priors for all parameters in SHMSRM-T.

The prior distributions for the other parameters in SHMSRM-T, such as $\boldsymbol{\beta}, \boldsymbol{\varrho}, \boldsymbol{\omega}$ and $\boldsymbol{\eta}$, are the same with the priors in SHMSRM-G. Therefore, the corresponding joint probability density function can be given by

$$
\begin{aligned}
p(\mathbf{Y}, \boldsymbol{\Xi})= & p(\mathbf{Y}) \prod_{m=1}^{\widetilde{M}} p\left(\varrho_{m}\right) p\left(\beta_{m}\right) \prod_{k=1}^{K} p\left(\eta_{k}\right) p\left(\omega_{k}\right) \\
& \cdot \prod_{i=1}^{N} p\left(\phi_{i}\right) p\left(\sigma_{i}\right) p\left(\nu_{i}\right)
\end{aligned}
$$

where $\phi=\left\{\phi_{1}, \cdots, \phi_{N}\right\}, \boldsymbol{\sigma}=\left\{\sigma_{1}, \cdots, \sigma_{N}\right\}, \boldsymbol{\nu}=$ $\left\{\nu_{1}, \cdots, \nu_{N}\right\}$, and $\boldsymbol{\Xi}=\{\boldsymbol{\varrho}, \boldsymbol{\beta}, \boldsymbol{\omega}, \boldsymbol{\eta}, \boldsymbol{\phi}, \boldsymbol{\sigma}, \boldsymbol{\nu}\}$. Then, our goal turns to infer the posteriors of all involved variables in (27).

2) Posterior distributions of all parameters in SHMSRM-T: VB is also employed to estimate all parameters in SHMSRM$\mathrm{T}$. The posterior of $\boldsymbol{\beta}$ in SHMSRM-T is also a multivariate Gaussian distribution, whose parameters are similar to (14). Its expression is

$$
\begin{gathered}
q(\boldsymbol{\beta})=\mathcal{N}\left(\boldsymbol{\beta} \mid \mu_{\boldsymbol{\beta}}, \boldsymbol{\Sigma}_{\boldsymbol{\beta}}\right), \\
\left.\boldsymbol{\Sigma}_{\boldsymbol{\beta}}=\left[\sum_{i=1}^{N}\left\langle\phi_{i}\right\rangle\left\langle\sigma_{i}^{-1}\right\rangle \widetilde{\mathbf{H}}^{(i, \cdot) \top}\left\langle\boldsymbol{\omega} \boldsymbol{\omega}^{\top}\right\rangle \widetilde{\mathbf{H}}^{(i, \cdot)}+\mathbf{A}\right)\right]^{-1}, \\
\boldsymbol{\mu}_{\boldsymbol{\beta}}=\sum_{i=1}^{N}\left\langle\phi_{i}\right\rangle\left\langle\sigma_{i}^{-1}\right\rangle y_{i} \boldsymbol{\Sigma}_{\boldsymbol{\beta}} \widetilde{\mathbf{H}}^{(i, \cdot) \top}\langle\boldsymbol{\omega}\rangle .
\end{gathered}
$$

As to the parameter $\omega$, its posterior distribution is

$$
\begin{gathered}
q(\boldsymbol{\omega})=\mathcal{N}\left(\boldsymbol{\omega} \mid \boldsymbol{\mu}_{\boldsymbol{\omega}}, \boldsymbol{\Sigma}_{\boldsymbol{\omega}}\right), \\
\boldsymbol{\Sigma}_{\boldsymbol{\omega}}=\left[\sum_{i=1}^{N}\left\langle\phi_{i}\right\rangle\left\langle\sigma_{i}^{-1}\right\rangle \widetilde{\mathbf{H}}^{(i, \cdot)}\left\langle\boldsymbol{\beta} \boldsymbol{\beta}^{\top}\right\rangle \widetilde{\mathbf{H}}^{(i, \cdot) \top}+\mathbf{B}\right]^{-1}, \\
\boldsymbol{\mu}_{\boldsymbol{\omega}}=\sum_{i=1}^{N}\left\langle\phi_{i}\right\rangle\left\langle\sigma_{i}^{-1}\right\rangle y_{i} \boldsymbol{\Sigma}_{\boldsymbol{\omega}} \widetilde{\mathbf{H}}^{(i, \cdot)}\langle\boldsymbol{\beta}\rangle .
\end{gathered}
$$

Taking the expectation of $\log p(\mathbf{Y}, \boldsymbol{\Xi})$ with respect to $\boldsymbol{\Xi}$ except for $\phi_{i}$, the posterior of $\phi_{i}$ is a Gamma distribution,

$$
\begin{gathered}
q\left(\phi_{i}\right)=\mathcal{G}\left(\phi_{i} \mid m_{\phi_{i}}, n_{\phi_{i}}\right) \\
m_{\phi_{i}}=\frac{\left\langle\nu_{i}\right\rangle}{2}+\frac{1}{2} \\
n_{\phi_{i}}=\frac{\left\langle\nu_{i}\right\rangle}{2}+\frac{1}{2}\left\langle\sigma_{i}^{-1}\right\rangle\left\langle\left(y_{i}-\boldsymbol{\omega}^{\top} \widetilde{\mathbf{H}}^{(i, \cdot)} \boldsymbol{\beta}\right)^{2}\right\rangle .
\end{gathered}
$$

For the parameter $\sigma_{i}$, its posterior distribution $q\left(\sigma_{i}\right)$ is an inverse Gamma distribution parameterized by $h_{\sigma_{i}}, i_{\sigma_{i}}$,

$$
\begin{gathered}
q\left(\sigma_{i}\right)=\mathcal{I} \mathcal{G}\left(\sigma_{i} \mid h_{\sigma_{i}}, i_{\sigma_{i}}\right), \\
h_{\phi_{i}}=h_{0}+\frac{1}{2}, \\
i_{\phi_{i}}=i_{0}+\frac{1}{2}\left\langle\phi_{i}\right\rangle\left\langle\left(y_{i}-\boldsymbol{\omega}^{\top} \widetilde{\mathbf{H}}^{(i, \cdot)} \boldsymbol{\beta}\right)^{2}\right\rangle .
\end{gathered}
$$

According to the expectation of $\log p(\mathbf{Y}, \boldsymbol{\Xi})$ with respect to $\boldsymbol{\Xi}$ except for $\nu_{i}$, the logarithmic posterior of $\nu_{i}$ can be expressed as (dropping the constant term)

$$
\begin{gathered}
\log q\left(\nu_{i}\right)=\frac{\nu_{i}}{2} \log \frac{\nu_{i}}{2}-\log \Gamma\left(\frac{\nu_{i}}{2}\right)-\frac{\nu_{i}}{2}\left\langle\phi_{i}\right\rangle+ \\
\left(\frac{\nu_{i}}{2}-1\right)\left\langle\log \phi_{i}\right\rangle+\left(j_{0}-1\right) \log \nu_{i}-k_{0} \nu_{i} .
\end{gathered}
$$

From (32), $q\left(\nu_{i}\right)$ does not obey any common distributions. According to the theory of Stirling's approximation, $\log \left(\nu_{i} / 2\right)$ can be approximated by the following equation [34],

$$
\log \Gamma\left(\frac{\nu_{i}}{2}\right)=\frac{1}{2}\left(\log 2 \pi-\log \frac{\nu_{i}}{2}\right)+\frac{\nu_{i}}{2}\left(\log \frac{\nu_{i}}{2}-1\right) .
$$

Therefore, it can be inferred that $q\left(\nu_{i}\right)$ is Gamma distributed by replacing the $\log \left(\nu_{i} / 2\right)$ in (32) by (33),

$$
q\left(\nu_{i}\right)=\mathcal{G}\left(\nu_{i} \mid r_{\nu_{i}}, s_{\nu_{i}}\right)
$$

where

$$
\begin{gathered}
r_{\nu_{k}}=r_{0}+\frac{1}{2}, \\
s_{\nu_{k}}=s_{0}-\frac{1}{2}\left[1+\left\langle\log \phi_{i}\right\rangle-\left\langle\phi_{i}\right\rangle\right] .
\end{gathered}
$$

As to the other parameters $\eta_{k}$ and $\varrho_{m}$, it can be seen from Fig. 1 and Fig. 2 that they are only related with their own priors and the priors of their related parameters $\left(\beta_{m}, \omega_{k}\right)$. Due to the priors for $\boldsymbol{\beta}, \varrho, \omega$ and $\boldsymbol{\eta}$ here are the same as the priors in SHMSRM-G. So, the posterior distributions of $\eta_{k}$ and $\varrho_{m}$ are the same with (19) and (15), respectively. The optimization procedure of SHMSRM-T is summarized in Algorithm 2.

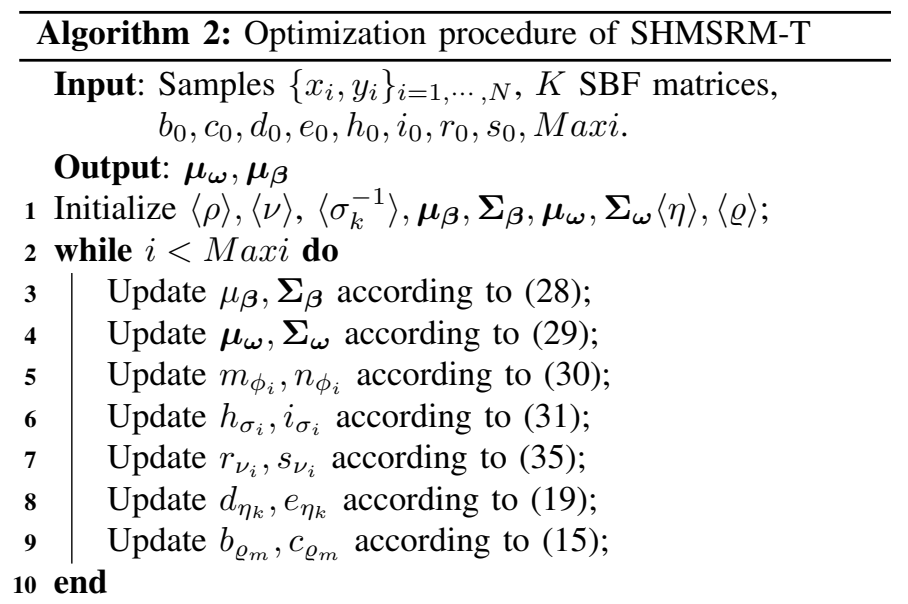

\section{PROCESSES OF WTPC MODELING}

Due to the complicated environment or a wind turbine fault, there are many outliers in the collected wind data. In general, an accurate WTPC cannot be obtained with the original unprocessed data. So, it is an essential step to preprocess the collected wind data [1] [12].

In this paper, a fuzzy c-means (FCM) clustering-based approach is used to detect outliers automatically. First, FCM is used to divide the data into $T$ clusters. The $i$ th sample in the $t$ th cluster can be expressed as $\boldsymbol{x}_{i}^{t}\left(i=1, \cdots, N_{t}, t=1, \cdots, T\right)$, 
TABLE I

DATA COLLECTED POSITION, NUMBER OF SAMPLES IN TRAINING AND TESTING SETS, AND VALUES OF SOME STATISTICS.

\begin{tabular}{|c|c|c|c|c|c|c|c|c|c|}
\hline Dataset & Latitude & Longitude & All samples & Training set & Testing set & Mean $(\mathrm{kW})$ & $\operatorname{Min}(\mathrm{kW})$ & $\operatorname{Max}(\mathrm{kW})$ & Std $(\mathrm{kW})$ \\
\hline A (Spring) & $\mathrm{N} 43^{\circ} 15^{\prime} 54^{\prime \prime}$ & E $117^{\circ} 32^{\prime} 44^{\prime \prime}$ & 6000 & 5000 & 1000 & 136.5125 & 0.0000 & 854.9500 & 217.4973 \\
\hline $\mathrm{B}$ (Winter) & $\mathrm{N} 43^{\circ} 15^{\prime} 54^{\prime \prime}$ & E $117^{\circ} 32^{\prime} 44^{\prime \prime}$ & 6000 & 5000 & 1000 & 244.8589 & 0.0000 & 856.9252 & 274.2028 \\
\hline $\mathrm{C}$ (Xichang) & $\mathrm{N} 42^{\circ} 55^{\prime} 56^{\prime \prime}$ & E $119^{\circ} 1^{\prime} 43^{\prime \prime}$ & 5500 & 4500 & 1000 & 459.3550 & 0.2527 & 1564.9000 & 428.0186 \\
\hline D (Hunan) & $\mathrm{N} 27^{\circ} 14^{\prime} 40^{\prime \prime}$ & E $113^{\circ} 10^{\prime} 54^{\prime \prime}$ & 9500 & 7500 & 2000 & 656.6667 & 0.0000 & 2069.9500 & 791.0424 \\
\hline
\end{tabular}

TABLE II

FIVE CLUSTER CENTERS GENERATED BY FCM ON FOUR DATASETS

\begin{tabular}{|c|ccccc|}
\hline Dataset & Cluster Center 1 & Cluster Center 2 & Cluster Center 3 & Cluster Center 4 & Cluster Center 5 \\
\hline A (Spring) & $(3.2111,12.6162)$ & $\left(6.1589,1.1904 \times 10^{2}\right)$ & $\left(8.2129,2.7816 \times 10^{2}\right)$ & $\left(10.9976,5.4984 \times 10^{2}\right)$ & $\left(14.8659,7.8673 \times 10^{2}\right)$ \\
B (Winter) & $(3.9793,19.8650)$ & $\left(6.8292,1.6493 \times 10^{2}\right)$ & $\left(8.7311,3.4846 \times 10^{2}\right)$ & $\left(10.8429,5.7656 \times 10^{2}\right)$ & $\left(14.4399,8.0324 \times 10^{2}\right)$ \\
C (Xichang) & $(3.3939,80.9016)$ & $\left(5.5498,2.6569 \times 10^{2}\right)$ & $\left(6.5433,4.2758 \times 10^{2}\right)$ & $\left(9.5472,1.0555 \times 10^{3}\right)$ & $\left(13.3308,1.4909 \times 10^{3}\right)$ \\
D (Hunan) & $(1.6113,13.5565)$ & $\left(4.3617,3.9997 \times 10^{2}\right)$ & $\left(6.2745,9.6424 \times 10^{2}\right)$ & $\left(7.7831,1.5132 \times 10^{3}\right)$ & $\left(10.4993,1.9761 \times 10^{3}\right)$ \\
\hline
\end{tabular}

and $\sum_{t=1}^{T} N_{t}=N$. Then, the mean $\boldsymbol{\mu}_{t}$ (also known as cluster center) and covariance $\boldsymbol{\Sigma}_{t}$ of all samples in the $t$ th cluster can be computed. Third, the Mahalanobis distance $d i s_{i}^{t}$ between the $t$ th cluster center $\boldsymbol{\mu}_{t}$ and the sample $\boldsymbol{x}_{i}^{t}$ should be calculated based on $d i s_{i}^{t}=\left(\boldsymbol{x}_{i}^{t}-\boldsymbol{\mu}_{t}\right) \boldsymbol{\Sigma}_{t}^{-1}\left(\boldsymbol{x}_{i}^{t}-\boldsymbol{\mu}_{t}\right)^{\top}$. Fourth, if the distance $d i s_{i}^{t}$ is larger than the given threshold, the sample $\boldsymbol{x}_{i}^{t}$ can be considered as an outlier; otherwise, it is a normal sample. Finally, the selected normal samples are used to the train the WTPC models, and get the final WTPCs.

\section{CAse Studies}

In this section, the performances of different WTPC models are evaluated in different seasons and different wind farms.

\section{A. Data description}

Saihanba wind farm, China, provided two datasets, Dataset A and Dataset B. They were all collected from the same wind turbine but in spring and winter, respectively. The other two datasets, Dataset C and Dataset D, were from Xichang and Hunan wind farms, China, respectively. For the first two datasets, all samples were recorded every one minute. But in the remaining two datasets, the time resolution of the data was ten minutes. Only two variables, wind speed and wind power, were measured and preserved in all datasets. The entire dataset was divided into two subsets, a training set and a testing set. The training set is used to train the selected WTPC model, while the testing set is utilized to evaluate the model performance. Table I shows the information of all datasets, including data collected positions, number of samples in training and testing sets, and values of some statistics (mean, minimum, maximum and standard deviation) for all wind power data in the whole dataset.

\section{B. Experiment setting}

To test the effectiveness of SHMSRM-G and SHMSRM-T, five integrated curve fitting models and ten AI-based models are employed as benchmark models. The former contain 3PLF, 4-PLF, 5-PLF, 6-PLF and MHTan. The later include GP,
SVM, ELM, ANFIS, SRM, weighted ELM (WELM) [35], HSRM, RSRM, MoAG-ASR and MoAEP-ASR.

As to the integrated curve fitting models, similar to [3], [4] and [12], the optimal model parameters can be optimized by the backtracking search optimization algorithm (BSA) ${ }^{1}$. In SVM, the RBF kernel function is selected, and the all model parameters are tuned by grid search. For ELM and WELM, the activation function is Sigmoid. And the optimal number of hidden layers is chosen from the set $\{10,20, \cdots, 180,200\}$. The optimization procures and initialized model parameters of HSRM, RSRM, MoAG-ASR and MoAEP-ASR are presented in [4] and [1]. As to spline regression-based models, two candidate SBFs are truncated power basis and Bspline basis, the candidate number of knots is in the set $\{2,4,6, \cdots, 20\}$, and the order of spline is set as 3 . The proposed two models take the above two SBFs and ten numbers of knots into consideration. Thus, there will be twenty SBF matrices, namely $K=20$. As to all hyperparameters $\left\{b_{0}, c_{0}, d_{0}, e_{0}, f_{0}, g_{0}, h_{0}, i_{0}, r_{0}, s_{0}\right\}$, they are set as 0.001 . And, the maximum number of iterations $M a x i$ is set as 50 .

Three error indicators, mean absolute error (MAE) [4], root mean squared error (RMSE) [20] [24] and normalized mean absolute percentage error (NMAPE) [3] [24], are used to measure the performances of different WTPC models. To make a fair model comparison, all models are trained with the same training set, and they all have only one input (wind speed) and one output (wind power).

\section{WTPC modeling}

Here, the results of data preprocessing and WTPC modeling are analyzed.

1) Data preprocessing: Outliers prevent obtaining more accurate WTPCs. It is essential to preprocess the original wind data. According to the content in section IV, a FCM-based method is used for detecting outliers in training sets automatically. Here, the number of clusters and the threshold are set as 5 and 15, respectively. For different datasets, five cluster centers, presented in Table II, can be obtained when sending

\footnotetext{
${ }^{1}$ https://www.mathworks.com/matlabcentral/fileexchange/44842backtracking-search-optimization-algorithm
} 
TABLE III

THE RESULTS OF SHMSRM-G, SHMSRM-T AND BENCHMARK MODELS.

\begin{tabular}{|c|c|c|c|c|c|c|c|c|c|c|c|c|}
\hline \multirow{2}{*}{ Models } & \multicolumn{3}{|c|}{ Dataset A (Spring) } & \multicolumn{3}{|c|}{ Dataset B (Winter) } & \multicolumn{3}{|c|}{ Dataset C (Xichang) } & \multicolumn{3}{|c|}{ Dataset D (Hunan) } \\
\hline & MAE(kW) & RMSE(kW) & NMAPE & MAE(kW) & RMSE(kW) & NMAPE & MAE $(\mathrm{kW})$ & RMSE(kW) & NMAPE & MAE(kW) & RMSE(kW) & NMAPE \\
\hline 3-PLF & 15.1827 & 19.1000 & 1.8946 & 20.4266 & 25.3246 & 2.4529 & 20.8619 & 31.0964 & 1.3331 & 49.5778 & 64.1729 & 2.4043 \\
\hline 4-PLF & 15.1971 & 19.1161 & 1.8964 & 20.5274 & 25.4023 & 2.4650 & 21.1574 & 31.1785 & 1.3520 & 49.8178 & 64.3210 & 2.4159 \\
\hline 5-PLF & 10.1283 & 16.4637 & 1.2639 & 14.2197 & 20.9155 & 1.7076 & 19.0592 & 30.6037 & 1.2179 & 37.2068 & 55.9600 & 1.8043 \\
\hline 6-PLF & 10.1337 & 16.1827 & 1.2646 & 14.9511 & 21.2848 & 1.7954 & 18.5268 & 30.2350 & 1.1839 & 40.6550 & 57.7892 & 1.9716 \\
\hline MTHan & 11.5151 & 16.7755 & 1.4370 & 14.9748 & 21.6661 & 1.7982 & 25.6294 & 38.9473 & 1.6378 & 42.9278 & 59.8989 & 2.0818 \\
\hline SVM & 9.7656 & 16.0846 & 1.2186 & 12.5110 & 20.4217 & 1.5024 & 17.1861 & 30.1403 & 1.0982 & 32.0216 & 52.9679 & 1.5529 \\
\hline GP & 9.7715 & 16.0860 & 1.2194 & 12.6274 & 20.3898 & 1.5164 & 17.4269 & 29.7732 & 1.1136 & 32.6556 & 53.1104 & 1.5836 \\
\hline ELM & 9.9923 & 16.6761 & 1.2469 & 12.7763 & 20.5045 & 1.5342 & 18.4905 & 30.3686 & 1.1816 & 32.0073 & 53.2754 & 1.5522 \\
\hline ANFIS & 11.1184 & 19.4247 & 1.3874 & 12.3891 & 20.4108 & 1.4877 & 19.4030 & 31.0595 & 1.2399 & 31.9720 & 52.9914 & 1.5505 \\
\hline SRM & 9.8720 & 16.5259 & 1.2319 & 12.8612 & 20.5692 & 1.5444 & 18.5585 & 30.6420 & 1.1859 & 34.5597 & 54.3409 & 1.6760 \\
\hline WELM & 9.8852 & 16.6264 & 1.2336 & 12.5660 & 20.2934 & 1.5090 & 16.8134 & 29.9042 & 1.0744 & 31.2720 & 52.8500 & 1.5165 \\
\hline HSRM & 9.7706 & 16.2107 & 1.2193 & 12.4909 & 20.1686 & 1.5000 & 16.5676 & 28.9852 & 1.0587 & 30.8560 & 52.1964 & 1.4964 \\
\hline RSRM & 9.7575 & 15.9058 & 1.2176 & 12.2733 & 20.3556 & 1.4738 & 16.4125 & 29.6338 & 1.0488 & 30.6879 & 53.3324 & 1.4882 \\
\hline MoAG-ASR & 9.8289 & 16.2620 & 1.2265 & 12.2699 & 19.9507 & 1.4734 & 16.3805 & 29.5380 & 1.0467 & 30.4604 & 54.6448 & 1.4772 \\
\hline MoAEP-ASR & 9.8199 & 16.1723 & 1.2254 & 12.2332 & 19.9879 & 1.4690 & 16.2176 & 29.6430 & 1.0363 & 30.4849 & 52.5489 & 1.4784 \\
\hline SHMSRM-G & 9.7473 & 16.0979 & 1.2164 & 12.0004 & 19.8486 & 1.4411 & 15.6231 & 28.7664 & 0.9983 & 30.6741 & 52.3980 & 1.4875 \\
\hline SHMSRM-T & 9.7402 & 16.0757 & 1.2155 & 12.1552 & 20.1066 & 1.4597 & 15.1624 & 28.6887 & 0.9689 & 29.8551 & 52.0828 & 1.4478 \\
\hline
\end{tabular}

the wind speed and wind power data into FCM. Meanwhile, all training samples can be classified into 5 clusters. Then, in each cluster, the Mahalanobis distance between the cluster center and the samples belonging to the cluster can be computed separately. Fig. 3 presents the calculated Mahalanobis distance in Cluster 1 and Cluster 5 of Dataset D. In Fig. 3, the red point represents an outlier, whose Mahalanobis distance is greater than the defined threshold 15. For different clusters, some outliers can be detected and removed. Finally, the processed wind data in Dataset A and Dataset D are presented in Fig. 4.
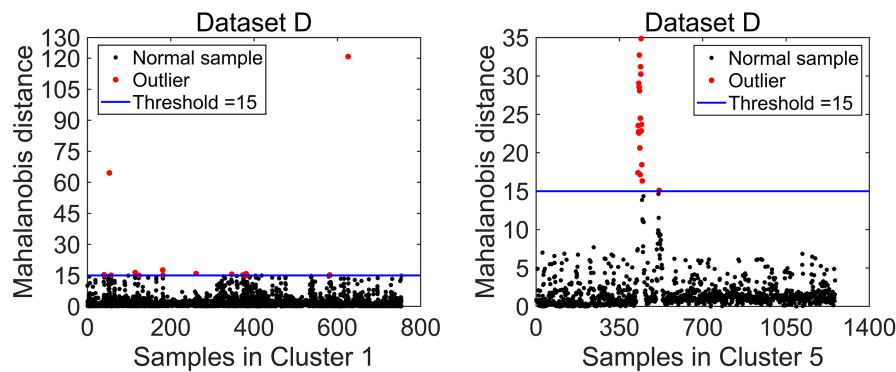

Fig. 3. The calculated Mahalanobis distance in Cluster 1 and Cluster 5.
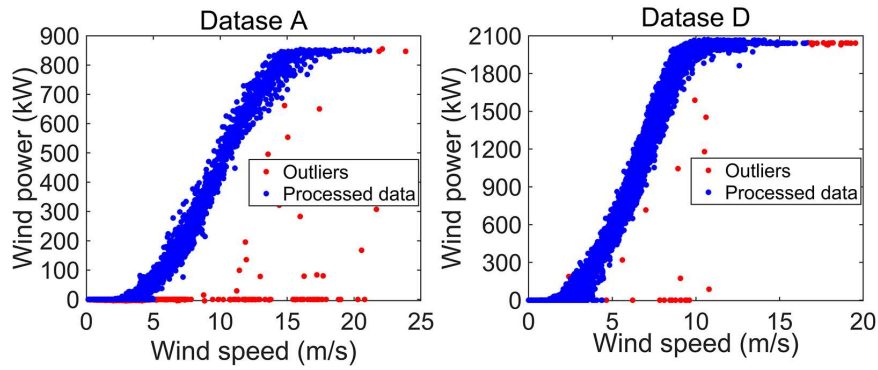

Fig. 4. The processed wind data and detected outliers.

From Fig. 4, most obvious outliers can be detected. But no one can grantee that the wind data preprocessing methods can recognize all outliers. Similar to [12], [13] and [24], there will be still some hidden outliers in the processed data. So, developing novel WTPC models that are robust to outliers is necessary.

2) Results of WTPC modeling: This paper proposes two novel WTPC models, which consider the characteristic of WTPC modeling in the presence of outliers. The processed wind data are used to train those WTPC models (including the proposed models and benchmark models), the model performances on testing set are summarized in Table III.

Among all benchmark models, the AI-based models can be divided into two groups. The first group consists of some popular WTPC models, e.g., GP, SVM, ELM, ANFIS and SRM. The remaining AI-based models compromise the second group. And the proposed models also belong the second group. All AI-based models in the second group can be considered as the models that consider the characteristic of WTPC modeling. Specifically, WELM, HSRM, SHMSRM-G and SHMSRM-T focus on the difference of estimated errors between samples, namely the heteroscedasticity, while RSRM, MoAG-ASR and MoAEP-ASR consider the overall distribution of estimated errors in all samples, namely the non-Gaussian characteristic.

From Table III, the majority of AI-based models perform better than the integrated curve fitting models in different datasets. In Dataset A, Dataset B and Dataset D, 5-PLF is the best integrated curve fitting model, while 6-PLF performs better than the other integrated curve fitting models in Dataset C. Generally, the models that consider the characteristics of WTPC modeling will outperform the models without considering these characteristics. So, among all AI-based benchmark models, it is not surprising to see that the majority of models in the second group are superior to the models in the first group. In all datasets, the best AI-based benchmark models are RSRM, MoAEP-ASR, MoAEP-ASR and MoAG-ASR, respectively, in terms of MAE and NMAPE.

There is no universal WTPC model that can always perform 
TABLE IV

AVERAGE IMPROVEMENT PERCENTAGES OF THE PROPOSED MODELS AND THE COMPUTATIONAL TIME OF ALL MODELS.

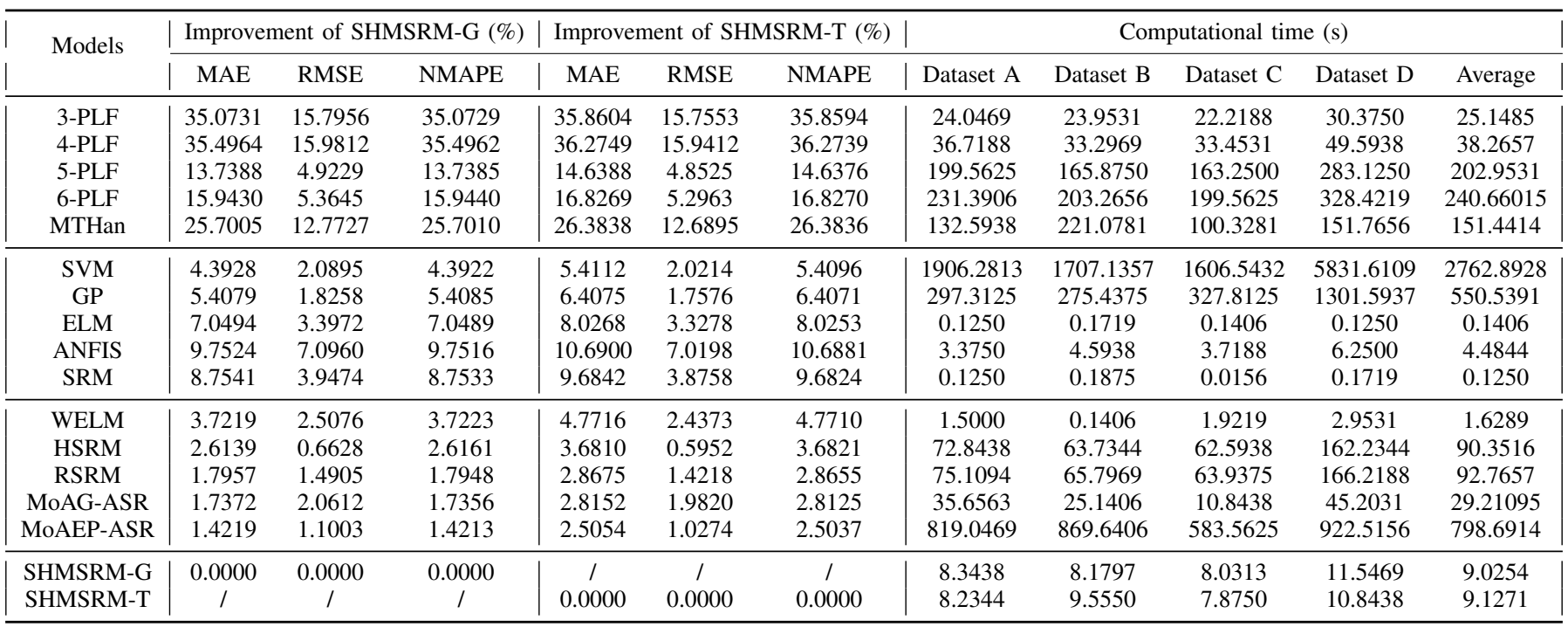

better than the others under different conditions. Overall, the proposed two models outperform the other benchmark models. In spring (Dataset A), in terms of MAE and NMAPE, SHMSRM-T performs the best among all WTPC models. But RSRM produces a WTPC with the lowest RMSE. For the same wind turbine in winter (Dataset B), SHMSRM-G is the best WTPC model. As for the wind turbines in Xichang (Dataset C) and Hunan (Dataset D) wind farms, SHMSRM-T is superior to all benchmark models and SHMSRM-G in terms of three error indicators.

In different datasets, compared with fifteen benchmark models, the improvement percentages of MAE, RMSE and NMAPE for SHMSRM-G and SHMSRM-T are computed, respectively, as shown in [36]. The average improvement percentages for all datasets are presented in Table IV. Generally, it can be found that, for the proposed models, the average improvement percentages of MAE and NMAPE reach the maximum and minimum when compared with 4-PLF and MoAEP-ASR, respectively. Compared with HSRM, the lowest average improvement percentages of RMSE can be obtained.
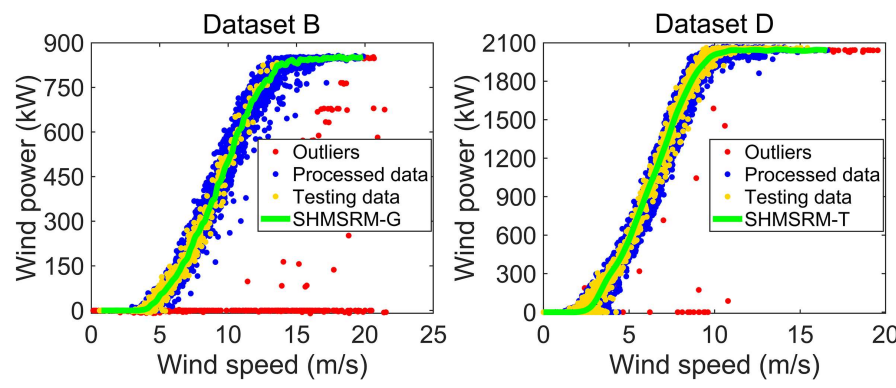

Fig. 5. The estimated WTPCs in Dataset B and Dataset D.

The estimated WTPCs generated by the optimal WTPC models in Dataset B and Dataset D are shown in Fig. 5. From Fig. 5, the estimated WTPCs can fit the wind data properly, thus describing the actual performances of wind turbines in practice. Moreover, different from the smooth ideal WTPC provided by manufacturer, the estimated actual WTPC may be a nonsmooth line, as can be seen in Dataset B.

\section{Discussion}

In terms of different error metrics, the ranks of all WTPC models can be computed. Then, the average ranks of different models on all datasets are employed to measure their overall performances in the task of WTPC modeling. The corresponding results are shown in Fig. 6.

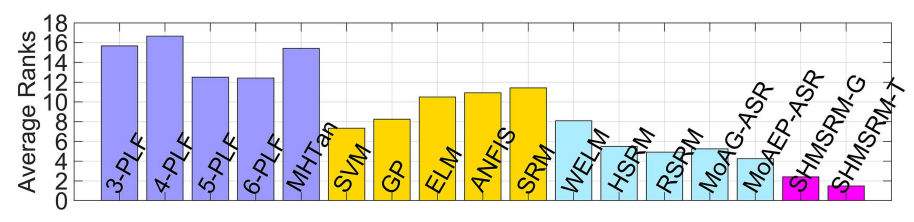

Fig. 6. Average ranks of WTPC models on all datasets.

From Fig. 6, among the seventeen models, SHMSRM-T and SHMSRM-G rank first and second, respectively, in WTPC modeling. All AI-based models rank higher than five integrated curve fitting benchmark models. The reason may be that the former is more flexible than the later, thus can simulate more complex WTPCs. In twelve AI-based models, except WELM, the models in the second group perform better than the models in the first group. This phenomenon indicates that developing novel regression models that consider the task characteristic of WTPC modeling can help generate more accurate WTPCs. The ranks of HSRM, RSRM, MoAG-ASR and MoAEP-ASR are close to each other. It shows that whether considering the heteroscedasticity or the non-Gaussian error distribution, the constructed regression models show the similar robust performance in the presence of outliers.

There are three major reasons why the performances of the proposed two models are better than that of the other benchmark models. First, consideration of the heteroscedastic 
characteristic of WTPC modeling, which is also the biggest difference among the proposed models, five integrated curve fitting models and the models in the first group of twelve AIbased methods. Second, utilization of multiple SBFs with different numbers of knots, which helps to describe the complex nonlinear relationship between wind speed and wind power. Third, the usage of sparse prior distributions on the regression coefficients and the weights of SBF matrices. The sparsity, as shown in Fig. 7, can help reduce the bad effects of some redundant SBFs and some useless mapping features.

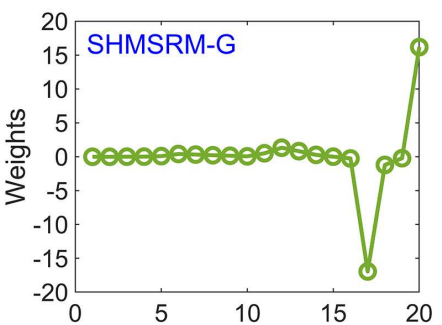

(a) Feature mapping functions

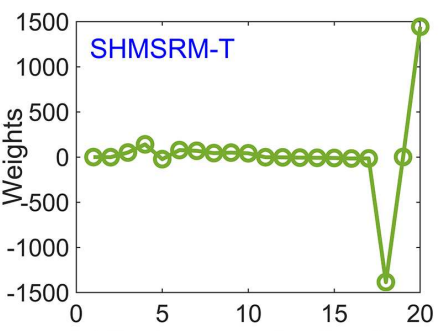

(c) Feature mapping functions

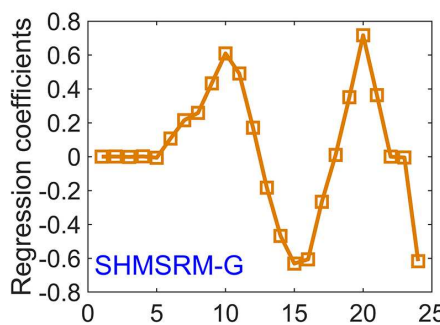

(b) Mapping features

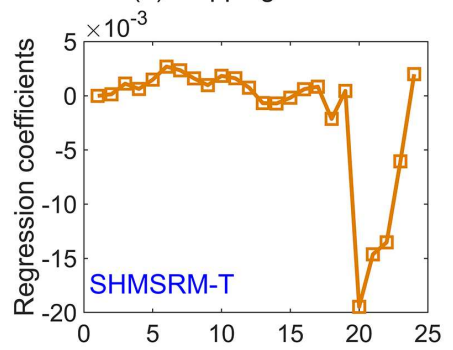

(d) Mapping features
Fig. 7. The estimated weights and regression coefficients on Dataset D.

From Fig. 7 (b) and (d), the closer the value of the regression coefficient is to 0 , the smaller the effect of the corresponding feature on the final regression results. Fig. 7 (a) and (c) show the similar result. Some values of weights are very close 0 , that means the corresponding SBFs have less effects on the final regression performance.

The computational time (CPU time) of different models is also compared. The results are shown in Table IV. It can be seen from Table IV that the computational time of the proposed models is less than the majority of compared models, including five integrated curve fitting models and six AI-based models. However, for the other four AI-based models (SRM, ELM, WELM and ANFIS), their computational time is less than the proposed models because they have fewer model parameters to be tuned. As to SVM and GP, they usually have higher computational complexity due to the large kernel matrix, thus resulting in more computational time. Compared with the other four spline-based regression models (HSRM, RSRM, MoAG-ASR and MoAEP-ASR), the advantage of the proposed models in computational time is also obvious. On the whole, the proposed models have higher power curve modeling accuracy with suitable computational time.

\section{CONCLUSIONS}

WTPC modeling is crucial in the development and utilization of wind energy. Some current WTPC models have the limited fitting ability because they do not consider the characteristic of WTPC modeling and they have the limited fitting ability in describing the complex nonlinear relationship between wind speed and wind power. To overcome these disadvantages, this paper proposes two novel WTPC models, SHMSRM$\mathrm{G}$ and SHMSRM-T. Compared with the performances of fifteen benchmark WTPC models in different seasons and different wind farms, the results show that (a) as long as the characteristics of WTPC modeling are considered, whether it is heteroscedasticity or non-Gaussian error distribution, the accuracy of WTPC modeling can be enhanced; (b) the actual WTPCs are not always smooth, they may be non-smooth under complex environmental conditions; (c) a combination of multiple spline regression models can help describe more complex nonlinear WTPCs than single models; (d) sparse prior distributions on regression coefficients and weights of SBF matrics can help select useful SBFs and mapping features. In addition to the high accuracy, the two proposed models also have high computational efficiency.

\section{REFERENCES}

[1] Y. Wang, Q. Hu, and S. Pei, "Wind power curve modeling with asymmetric error distribution," IEEE Trans. Sustain. Energy, 2019.

[2] T. Jin and Z. Tian, "Uncertainty analysis for wind energy production with dynamic power curves," in IEEE 11th Int. Conf. Probabilistic Methods Appl. Power Syst, 2010, pp. 745-750.

[3] Y. Wang, Q. Hu, L. Li, A. M. Foley, and D. Srinivasan, "Approaches to wind power curve modeling: A review and discussion," Renewable Sustain. Energy Rev., vol. 116, p. 109422, 2019.

[4] Y. Wang, Q. Hu, D. Srinivasan, and Z. Wang, "Wind power curve modeling and wind power forecasting with inconsistent data," IEEE Trans. Sustain. Energy, vol. 10, no. 1, pp. 16-25, 2018.

[5] S. Mathew, Wind energy: fundamentals, resource analysis and economics. Springer, 2006, vol. 1.

[6] B. Ai, H. Yang, H. Shen, and X. Liao, "Computer-aided design of pv/wind hybrid system," Renewable Energy, vol. 28, no. 10, pp. 14911512, 2003.

[7] Y. Yan, L. A. Osadciw, G. Benson, and E. White, "Inverse data transformation for change detection in wind turbine diagnostics," in 2009 IEEE CCECE, 2009, pp. 944-949.

[8] L. A. Osadciw, Y. Yan, X. Ye, G. Benson, and E. White, "Wind turbine diagnostics based on power curve using particle swarm optimization," in Wind Power Systems. Springer, 2010, pp. 151-165.

[9] Q. Hernández-Escobedo, F. Manzano-Agugliaro, and A. Zapata-Sierra, "The wind power of mexico," Renewable Sustain. Energy Rev., vol. 14, no. 9 , pp. 2830-2840, 2010.

[10] M. Lydia, A. I. Selvakumar, S. S. Kumar, and G. E. P. Kumar, "Advanced algorithms for wind turbine power curve modeling," IEEE Trans. Sustain. Energy, vol. 4, no. 3, pp. 827-835, 2013.

[11] D. Villanueva and A. Feijóo, "Comparison of logistic functions for modeling wind turbine power curves," Electr. Pow. Syst. Res., vol. 155, pp. 281-288, 2018.

[12] E. Taslimi-Renani, M. Modiri-Delshad, M. F. M. Elias, and N. A. Rahim, "Development of an enhanced parametric model for wind turbine power curve," Appl. Energy, vol. 177, pp. 544-552, 2016.

[13] T. Ouyang, A. Kusiak, and Y. He, "Modeling wind-turbine power curve: A data partitioning and mining approach," Renewable Energy, vol. 102, pp. 1-8, 2017.

[14] R. K. Pandit and D. Infield, "Scada-based wind turbine anomaly detection using gaussian process models for wind turbine condition monitoring purposes," IET Renew. Power Gen., vol. 12, no. 11, pp. 1249-1255, 2018.

[15] S. Pei and Y. Li, "Wind turbine power curve modeling with a hybrid machine learning technique," Appl. Sci., vol. 9, no. 22, p. 4930, 2019.

[16] M. Schlechtingen, I. F. Santos, and S. Achiche, "Using data-mining approaches for wind turbine power curve monitoring: a comparative study," IEEE Trans. Sustain. Energy, vol. 4, no. 3, pp. 671-679, 2013.

[17] M. Mehrjoo, M. J. Jozani, and M. Pawlak, "Wind turbine power curve modeling for reliable power prediction using monotonic regression," Renewable Energy, vol. 147, pp. 214-222, 2020. 
[18] B. Stephen, S. J. Galloway, D. McMillan, D. C. Hill, and D. G. Infield, "A copula model of wind turbine performance," IEEE Trans. Power Syst., vol. 26, no. 2, pp. 965-966, 2010.

[19] Y. Zhao, L. Ye, W. Wang, H. Sun, Y. Ju, and Y. Tang, "Data-driven correction approach to refine power curve of wind farm under wind curtailment," IEEE Trans. Sustain. Energy, vol. 9, pp. 95-105, 2017.

[20] M. Yesilbudak, "Implementation of novel hybrid approaches for power curve modeling of wind turbines," Energy Convers. Manage., vol. 171, pp. 156-169, 2018.

[21] T. Rogers, P. Gardner, N. Dervilis, K. Worden, A. Maguire, E. Papatheou, and E. Cross, "Probabilistic modelling of wind turbine power curves with application of heteroscedastic Gaussian Process regression," Renewable Energy, vol. 148, pp. 1124-1136, 2020.

[22] A. P. Dempster, N. M. Laird, and D. B. Rubin, "Maximum likelihood from incomplete data via the EM algorithm," Journal of the Royal Statistical Society: Series B, vol. 39, no. 1, pp. 1-22, 1977.

[23] C. M. Bishop, Pattern Recognition and Machine Learning. Springer, 2006.

[24] S. Shokrzadeh, M. J. Jozani, and E. Bibeau, "Wind turbine power curve modeling using advanced parametric and nonparametric methods," IEEE Trans. Sustain. Energy, vol. 5, no. 4, pp. 1262-1269, 2014.

[25] M. Zhou, H. Chen, L. Ren, G. Sapiro, L. Carin, and J. W. Paisley, "Nonparametric Bayesian dictionary learning for sparse image representations," in Neural Information Processing Systems, 2009, pp. 2295-2303.

[26] B. A. Olshausen and D. J. Field, "Sparse coding with an overcomplete basis set: A strategy employed by v1?" Vision research, vol. 37, no. 23, pp. 3311-3325, 1997

[27] D. P. Wipf, "Bayesian methods for finding sparse representations," Ph.D. dissertation, UC San Diego, 2006.

[28] T. Buchgraber, "Variational sparse Bayesian learning: Centralized and distributed processing," Ph.D. dissertation, Graz University of Technology, 2013.

[29] M. E. Tipping, "Sparse Bayesian learning and the relevance vector machine," J. Mach. Learn. Res., vol. 1, pp. 211-244, 2001.

[30] K. P. Murphy, Machine learning: a probabilistic perspective. MIT press, 2012

[31] T. M. Nguyen and Q. J. Wu, "Bounded asymmetrical student's-t mixture model," IEEE Trans. on Cybern., vol. 44, no. 6, pp. 857-869, 2013.

[32] K. Ning, M. Liu, and M. Dong, "A new robust ELM method based on a Bayesian framework with heavy-tailed distribution and weighted likelihood function," Neurocomputing, vol. 149, pp. 891-903, 2015.

[33] X. Wei and Z. Yang, "The infinite student's t-factor mixture analyzer for robust clustering and classification," Pattern Recogn., vol. 45, no. 12, pp. 4346-4357, 2012

[34] J. Christmas and R. Everson, "Robust autoregression: Student-t innovations using variational Bayes," IEEE Trans. Signal Process., vol. 59, no. 1 , pp. 48-57, 2011.

[35] W. Deng, Q. Zheng, and L. Chen, "Regularized extreme learning machine," in IEEE Symp. Comput. Intell. Data Mining, 2009, pp. 389395.

[36] Z. Liu, P. Jiang, L. Zhang, and X. Niu, "A combined forecasting model for time series: Application to short-term wind speed forecasting," Appl. Energy, vol. 259, p. 114137, 2020.

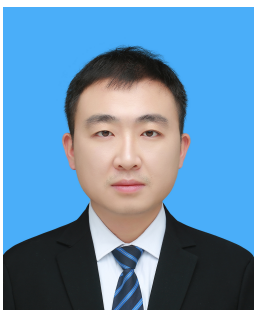

Yun Wang received the B.S., M.S. and Ph.D. degrees from Anhui University, Lanzhou University, Tianjin University, China, in 2012, 2015, 2019, respectively. Now, he is an associate professor in the School of Automation, Central South University, Changsha, China. His interests include wind speed/wind power forecasting, big data analysis in power systems, robust regression modeling, multi-kernel learning, functional data analysis, and Bayesian inference.

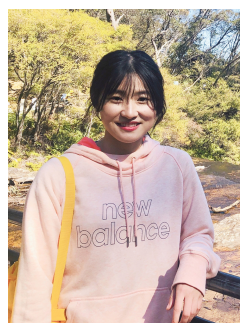

Yifen Li Yifen Li obtained the M.S. degree from the School of Traffic and Transportation, Changsha University of Science and Technology in 2014, and is currently a Ph.D. candidate in Traffic and Transportation Engineering at Central South University from 2017. She joined the Department of Automotive Engineering at Changsha Vocational and Technical College and became lecturer in 2018. Her research interests include rail transit safety, optimization methods, artificial intelligence.

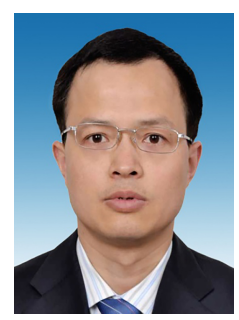

Runmin Zou received the Ph.D. degree in automatic control from the Ecole Centrale de Nantes, France, in 2009. He has been professor with School of Automation, Central South University, Changsha, China, since 2012. His main research interests lie in the intersection of control theory, machine learning and big data analysis, with their applications to renewable energy and complex dynamical systems.

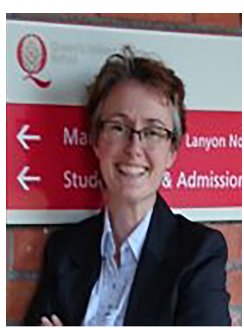

Aoife M. Foley received the B.Eng. (Hons.) and $\mathrm{Ph} . \mathrm{D}$. degrees from University College Cork, Ireland, in 1996 and 2011, respectively, and an M.Sc. degree from Trinity College, Ireland, in 1999. She worked in industry until 2008. She is currently a Lecturer in the School of Mechanical and Aerospace Engineering, Queens University Belfast, UK. Her research interests include wind energy, energy market$\mathrm{s}$, and electric vehicles. She is a Chartered Engineer (2001) and a Fellow of Engineers Ireland (2012).

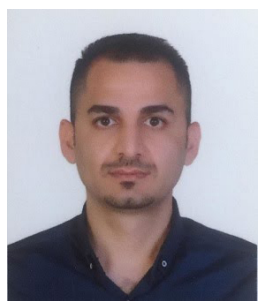

Dlzar Al Kez received the B.Sc. degree from University of Sulaimani, Iraq, in 2009, and the M.Sc. degree from University of Southampton, UK, in 2012. He is currently working toward the Ph.D. degree in Queen's University Belfast, UK. His interests include impact of distributed generation on the power system dynamics, impact of renewable energy on the power system dynamics.

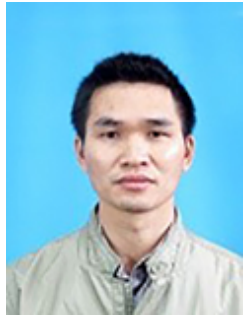

Dongran Song received the B.S., M.S. and Ph.D. degrees from Central South University, China, in 2006, 2009 and 2016, respectively, where he has been as an associate professor since 2018. He was a senior engineer with China Ming Yang Wind Power, Zhongshan, from 2009 to 2017, where he took part in the control and electrical system design of $1.5 \mathrm{MW}-6.0 \mathrm{MW}$ series wind turbines. His research interests include wind turbine control, power electronics control, and renewable energy system modeling and design.

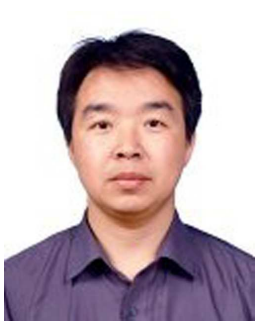

Qinghua Hu received the B.S., M.S., and Ph.D. degrees from the Harbin Institute of Technology, Harbin, China, in 1999, 2002, and 2008, respectively. He was a Postdoctoral Fellow with the Department of Computing, Hong Kong Polytechnic University, Hong Kong, from 2009 to 2011. He is currently a Full Professor and the Vice Dean of the College of Intelligence and Computing, Tianjin University, Tianjin, China. He has authored more than 100 journal and conference papers in the areas of granular computing based machine learning, reasoning with uncertainty, pattern recognition, and fault diagnosis. His current research interests include rough sets, granular computing, and data mining for classification and regression.

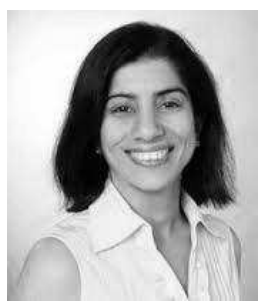

Dipti Srinivasan (M'89-SM'02) received the Ph.D degree in engineering from the National University of Singapore, Singapore, in 1994. She was a Postdoctoral Researcher with the University of California, Berkeley, Berkeley, CA, USA, from 1994 to 1995, before joining the National University of Singapore, where she is currently a Professor with the Department of Electrical and Computer Engineering. Her research interest include the application of soft computing techniques to engineering optimization and control problems. 